\title{
Suicidal drug overdose following stroke in elderly patients: a retrospective population-based cohort study
}

This article was published in the following Dove Press journal: Neuropsychiatric Disease and Treatment

\author{
Chun-Hung Chang ${ }^{1,2}$ \\ Shaw-Ji Chen ${ }^{3,4}$ \\ Chieh-Yu Liu ${ }^{5}$ \\ Hsin-Chi Tsai ${ }^{6,7}$
}

'Brain Disease Research Center, Department of Psychiatry, China Medical University Hospital, Taichung, Taiwan; ${ }^{2}$ Institute of Clinical Medical Science, China Medical University, Taichung, Taiwan; ${ }^{3}$ Department of Psychiatry, Mackay Memorial Hospital Taitung Branch, Taitung, Taiwan; ${ }^{4}$ Mackay Junior College of Medicine, Nursing and Management, Taipei, Taiwan; ${ }^{5}$ Biostatistical Consulting Lab, Institute of Nursing-Midwifery, National Taipei University of Nursing and Health Sciences, Taipei, Taiwan; ${ }^{6}$ Department of Psychiatry, Tzu-Chi General Hospital, Hualien, Taiwan; ${ }^{7}$ Institute of Medical Sciences, Tzu Chi University, Hualien, Taiwan
Correspondence: Hsin-Chi Tsai Department of Psychiatry, Tzu-Chi General Hospital, Number 707, Section 3, Chung Yang Road, Hualien 970, Taiwan

Tel +88638561825

Fax +88638574049

Email css30bmw@yahoo.com.tw
Purpose: The purpose of this study was to investigate the incidence and risk of suicidal drug overdose (SDO) after stroke in older patients.

Methods: We enrolled patients aged 60-99 years who were diagnosed with new-onset stroke between 2002 and 2013 and age-, sex-, and index-year-matched controls who did not have stroke. Patients with a history of SDO before enrollment were excluded. Both groups were observed until December 31, 2013. The primary end point was the occurrence of newly diagnosed SDO. The cumulative incidence rates of the study and control groups were estimated using the Kaplan-Meier method. Furthermore, we used the Cox proportional hazards model to identify risk factors for SDO.

Results: We selected 22,770 individuals. Among them, 11,385 were older patients (aged 60-99 years) who had newly diagnosed stroke and 11,385 were controls. Of the 22,770 individuals, 275 (1.21\%) had SDO during a mean follow-up period of $5.33 \pm 3.30$ years, comprising $191(1.68 \%)$ from the stroke group and $84(0.74 \%)$ from the control group. Older patients with stroke had a significantly higher risk of SDO than the controls (adjusted hazard ratio: 2.288, 95\% confidence interval [CI]: 1.746-2.999, $p<0.001)$. Moreover, in older patients with stroke, the risk significantly increased with the number of stroke events. Patients with depressive disorder or coronary disease had an increased risk of SDO. Additionally, benzodiazepines and anticoagulants were the two most commonly prescribed medications for SDO.

Conclusion: Clinicians should be aware of the risk of SDO and risk factors in older patients with stroke. Psychological assessment and medication monitoring should be incorporated into current clinical diagnoses in neurology and treatments following stroke.

Keywords: stroke, older people, suicidal drug overdose

\section{Introduction}

Stroke is an acute episode of focal dysfunction of neural organs, including the brain, retina, or spinal cord, lasting longer than 24 hours; stroke is also diagnosed if brain imaging or autopsy reveals focal infarction or hemorrhage. ${ }^{1}$ Stroke is the second leading cause of death and the third leading cause of disability worldwide. ${ }^{2,3}$ Studies have reported that patients with stroke exhibit an increased risk of suicide. ${ }^{4-6}$ A literature review indicated that 12 observational studies investigated the relationship between stroke and suicide ideation or planning ${ }^{7-17}$ and 11 observational studies evaluated suicide as a cause of death after stroke. ${ }^{18-28}$

However, a few studies have reported the long-term risk of suicide attempts following stroke. A study of 220,336 patients with stroke documented 1,217 suicide attempts from 2001 to 2012, and an increased risk of attempted suicide was associated 
with lower education or income, living alone, the male sex, young age, severe stroke, and poststroke depression. ${ }^{29}$ However, this trial investigated only the stroke population; the difference in the risk of suicide attempts between older patients with stroke and those without stroke remains unclear. Moreover, previous studies have shown that patients with stroke usually use nonviolent suicide methods (eg, selfpoisoning) rather than violent suicide methods (eg, hanging or shooting). ${ }^{4,30}$ Drug overdose is the most common nonviolent suicide method used. ${ }^{30,31}$ However, a few studies have examined the incidence and characteristics of suicidal drug overdose (SDO) after stroke in older patients; ${ }^{4}$ in addition, whether the risk of SDO is higher in these patients remains uncertain.

In the present study, we investigated the incidence of SDO after stroke in older patients. The results may guide future care strategies for stroke and suicide prevention programs for older patients.

\section{Methods}

\section{Data source}

The Taiwanese government launched the National Health Insurance (NHI) program in March 1, 1995. This program currently covers $>98.29 \%$ of the residents of Taiwan. ${ }^{32}$ Comprehensive information, including prescription details, clinic visits, and diagnostic codes, is recorded in the National Health Insurance Research Database (NHIRD). The diagnostic codes in the NHIRD are based on the International Classification of Diseases, Ninth Revision, Clinical Modification (ICD-9-CM).

\section{Ethics statement}

This study was approved by the institutional review board (IRB) of China Medical University Hospital (CMUH105REC2-087). Because information in the database that could identify any individual patient was encrypted by the Ministry of Health and Welfare of Taiwan, this study was exempted from the requirement to obtain written consent from the study patients by the IRB of China Medical University Hospital.

\section{Study sample and control}

In this study, older patients aged 60-99 years with newly diagnosed stroke (ICD-9-CM codes 430-5.x) from 2002 to 2013 were included in the study group (stroke group). Those with a history of stroke before January 1, 2002, were excluded to ensure that only patients with a first diagnosis of stroke were included. We excluded stroke patients with a history of SDO. To improve diagnostic validity, only patients who were admitted with stroke as the primary diagnosis were included in this study.

\section{Matching}

The control group of patients without stroke was selected at a ratio of one control patient to one patient with stroke. The control group was matched for each individual's age, sex, and enrollment year. Matching for age and year of enrollment was limited to the same age and year. The start date of follow-up was defined as the date of the first clinical visit to a medical facility in the enrollment year. All patients were observed until the occurrence of SDO, death, withdrawal from the NHI program, or December 31, 2013 (Figure 1).

\section{Main outcome measure}

The outcome measure of this study was the occurrence of SDO. SDO or self-poisoning was defined as an emergency department visit, a clinic visit, or hospitalization with the ICD-9 CM codes 960-979 but without E codes such as E850-E858 (accidental poisoning by drugs, medicinal substances, and biologicals). ${ }^{33-35}$ We excluded all E codes to increase diagnosis accuracy. Both groups were observed until the first occurrence of SDO, death, withdrawal from the NHI program, or December 31, 2013.

\section{Variables}

In this study, demographic data including age, sex, and index year were retrieved and matched between the two groups. In previous studies, ${ }^{4}$ risk factors for SDO and major comorbidities, including insomnia, depressive disorder, dementia, substance use disorders, alcohol use disorders, bipolar disorders, and psychotic disorders, have been assessed. In Taiwan, diagnoses for depressive disorders, bipolar disorders, or psychotic disorders are made in accordance with ICD-9 CM codes and the Diagnostic and Statistical Manual of Mental Disorders-IV by board-certified psychiatrists and physicians. In this study, only those having at least three consecutive corresponding diagnoses were designated as having depressive disorders for enhanced diagnostic validity. We also used the Charlson Comorbidity Index (CCI) score to evaluate baseline comorbidities.

\section{Statistical analysis}

The independent-samples $t$-test was used to compare age, follow-up period, and CCI scores between the two groups. The chi-square test was used to compare categorical variables such as age group, sex, and major comorbidities. The cumulative incidence rates of the study and control groups were estimated using the Kaplan-Meier method. To determine the 


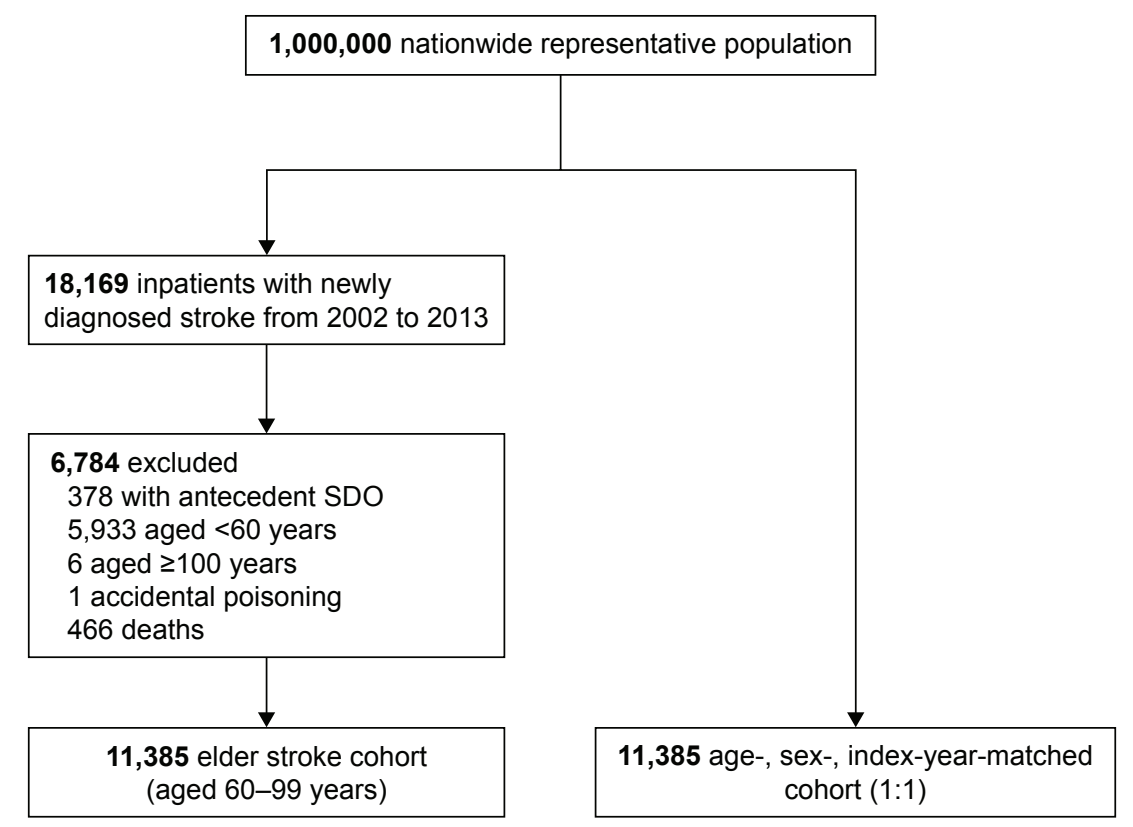

Figure I Flow diagram summarizing the process of enrollment and follow-up. Abbreviation: SDO, suicidal drug overdose.

independent risk factors for SDO, univariate and multivariate analyses using hazard ratios (HRs) were conducted with Cox proportional hazards models after adjustment for age, sex, mental health diagnosis, and major coexisting diseases. A sensitivity analysis in patients with 6-month follow-up was performed. MySQL was adopted for extracting, linking, and processing the study data. All statistical analyses were performed using IBM SPSS statistical software (version 19.0 for Windows; IBM Corporation, Armonk, NY, USA). A two-tailed $p$-value of $<0.05$ was considered as statistically significant.

\section{Results \\ Demographic characteristics of the stroke group}

A total of 11,385 older patients (aged 60-99 years) who had a first diagnosis of stroke from January 1, 2002, to December 31, 2013, and 11,385 matched controls (without stroke) were enrolled. The baseline characteristics of the stroke and control groups are listed in Table 1. The stroke group had a higher prevalence of comorbidities and higher CCI scores than the control group.

\section{Cumulative incidence of SDO}

During a mean follow-up period of $5.33 \pm 3.30$ years, the incidence of SDO was higher in the stroke group than in the control group $(3.31 / 1,000$ person-years vs $1.32 / 1,000$ person-years; Table 2). Older patients with stroke had a significantly higher risk of incident SDO than the controls (log-rank test, $p<0.001$; Figure 2). The adjusted hazard ratio (aHR) was 2.288 (95\% confidence interval [CI]: 1.746-2.999, $p<0.001$; Table 2). Furthermore, patients with more stroke events had a higher incidence of SDO (Figure 3).

\section{Risk factors for SDO in the stroke group}

Cox multivariate proportional hazard analysis showed that in older patients with stroke, depressive disorder (aHR: 1.543, 95\% CI: 1.001-2.378, $p=0.050)$ and coronary disease (aHR: 1.617, 95\% CI: 1.193-2.913, $p=0.002$ ) were independent risk factors for SDO (Table 3). Furthermore, we divided patients into four groups: the poststroke depression, prestroke depression, stroke without depression, and nonstroke control groups. We found that the poststroke depression group had the highest risk of SDO. The cumulative incidence rates were $4.9 \%, 3.7 \%, 2.7 \%$, and $1.4 \%$ for these groups, respectively (Figure 4).

\section{SDO analysis}

The top three drug classifications according to ICD-9-CM codes were 977.9 (poisoning by unspecified drug or medicinal substance, $21.47 \%, 41$ of 191 SDO events caused by unknown drugs), 969.4 (poisoning by benzodiazepine-based tranquilizers, $15.18 \%, 29$ of 191 SDO events caused by benzodiazepines), and 964.2 (poisoning by anticoagulants, $15.18 \%, 29$ of 191 SDO events caused by anticoagulants). 
Table I Demographic profile of study patients $(\mathrm{N}=22,770)$

\begin{tabular}{|c|c|c|c|c|}
\hline \multirow[t]{2}{*}{ Variables } & \multirow{2}{*}{$\begin{array}{l}\text { Stroke } \\
(n=\mid 1,385)\end{array}$} & \multirow{2}{*}{$\begin{array}{l}\text { Nonstroke } \\
(n=I I, 385)\end{array}$} & \multirow[t]{2}{*}{$t / \chi^{2}$} & \multirow[t]{2}{*}{$\overline{p \text {-value }}$} \\
\hline & & & & \\
\hline Age (years) & $73.4 I \pm 8.18$ & $73.4 I \pm 8.18$ & 0.000 & 1.000 \\
\hline $60-69$ & $4,024(35.3)$ & $4,024(35.3)$ & 0.000 & 1.000 \\
\hline $70-79$ & $4,527(39.8)$ & $4,527(39.8)$ & & \\
\hline $80-89$ & $2,53 \mid(22.1)$ & $2,531(22.1)$ & & \\
\hline $90-99$ & $303(2.7)$ & $303(2.7)$ & & \\
\hline Men & $6,234(54.8)$ & $6,234(54.8)$ & 0.000 & 1.000 \\
\hline Follow-up (years) & $5.06 \pm 3.30$ & $5.60 \pm 3.29$ & -12.352 & $<0.001$ \\
\hline \multicolumn{5}{|l|}{ Mental health diagnosis } \\
\hline Insomnia & $2,659(23.4)$ & $2,310(20.3)$ & 31.355 & $<0.00$ I \\
\hline Depressive disorders & I,047 (9.2) & $875(7.7)$ & 16.811 & $<0.001$ \\
\hline Dementia & $989(8.7)$ & $568(5.0)$ & 122.190 & $<0.001$ \\
\hline Substance use disorders & $175(1.5)$ & III (I.0) & 14.504 & $<0.001$ \\
\hline Alcohol use disorders & $226(2.0)$ & $89(0.8)$ & 60.420 & $<0.001$ \\
\hline Bipolar disorders & $68(0.6)$ & $58(0.5)$ & 0.798 & 0.372 \\
\hline Psychotic disorders & $59(0.5)$ & $49(0.4)$ & 0.930 & 0.335 \\
\hline \multicolumn{5}{|l|}{ Major coexisting diseases } \\
\hline Hypertension & $10,075(88.5)$ & $7,053(61.9)$ & $2,151.85 \mid$ & $<0.00$ I \\
\hline Diabetes & $4,960(43.6)$ & $3,044(26.7)$ & 707.268 & $<0.001$ \\
\hline Coronary disease & $5,091(44.7)$ & $3,753(33.0)$ & 330.979 & $<0.001$ \\
\hline COPD & $4,933(43.3)$ & 4,05 I (35.6) & 143.018 & $<0.001$ \\
\hline Chronic kidney disease & $2,696(23.7)$ & $1,916(16.8)$ & 165.423 & $<0.00$ I \\
\hline Asthma & I,950 (I7.I) & $\mathrm{I}, 484(\mathrm{I} 3.0)$ & 74.468 & $<0.001$ \\
\hline Fracture & $2,79 \mid(24.5)$ & $2,357(20.7)$ & 47.277 & $<0.00$ I \\
\hline Osteoporosis & $2,446(21.5)$ & $2,170(19.1)$ & 20.699 & $<0.001$ \\
\hline Hyperlipidemia & $2,792(24.5)$ & $2,028(17.8)$ & 153.617 & $<0.00$ I \\
\hline Charlson comorbidity score & $1.42 \pm 1.61$ & $0.75 \pm I .44$ & 32.856 & $<0.001$ \\
\hline
\end{tabular}

Note: Data shown as mean \pm standard deviation or $\mathrm{n}(\%)$.

Abbreviation: COPD, chronic obstructive pulmonary disease.

The results showed that most SDO events occurred within 1 year following stroke in older patients with stroke $(66 / 191$, 34.6\%; Figure 5). Moreover, a large proportion (174/191) of these patients had ischemic stroke. After adjustment for age and sex, older patients with ischemic stroke had a higher risk of SDO than those with hemorrhagic stroke (aHR: 1.8841, 95\% CI: $1.1446-3.1015, p=0.0127)$.

Table 2 Incidences and HRs for SDO following stroke (2002-20I2, N=22,770)

\begin{tabular}{llll}
\hline SDO during follow-up & $\begin{array}{l}\text { Total } \\
\text { sample }\end{array}$ & $\begin{array}{l}\text { Comparison } \\
\text { group }\end{array}$ & $\begin{array}{l}\text { Stroke } \\
\text { group }\end{array}$ \\
\hline $\begin{array}{l}\text { Incidence of stroke } \\
\text { (per I,000 person-years) }\end{array}$ & 2.64 & 1.32 & 3.31 \\
No of occurrences & 275 & 84 & 191 \\
Observed person-years & $121,416.75$ & $63,779.68$ & $57,637.07$ \\
Crude HR $(95 \% \mathrm{Cl})$ & & 1.00 & $\begin{array}{l}2.476 \\
(1.915-3.200)^{*}\end{array}$ \\
aHR $(95 \% \mathrm{Cl})^{\mathrm{a}}$ & & & 2.288 \\
& & 1.00 & $(1.746-2.999)^{*}$ \\
\hline
\end{tabular}

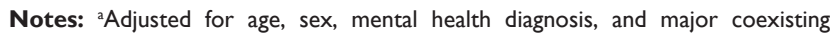
diseases. $* p<0.001$.

Abbreviations: HR, hazard ratio; SDO, suicidal drug overdose; $\mathrm{Cl}$, confidence interval; aHR, adjusted hazard ratio.

\section{Discussion}

The results of this nationwide population-representative cohort study show that 1) older patients with stroke had an increased risk of SDO; 2) the risk of SDO increased with the number of stroke events; 3 ) depressive disorder and coronary disease were independent risk factors for SDO;

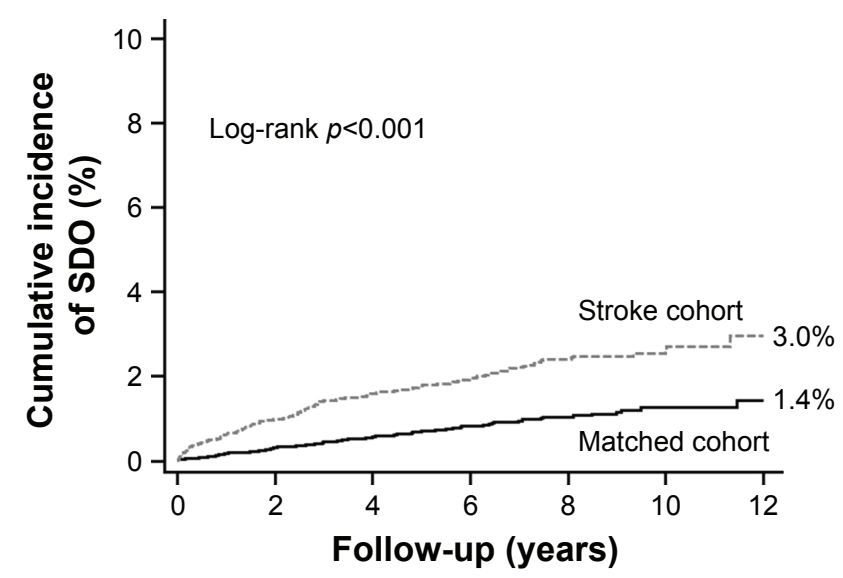

Figure 2 Cumulative incidence of SDO in elder patients with stroke and the matched cohort.

Abbreviation: SDO, suicidal drug overdose. 


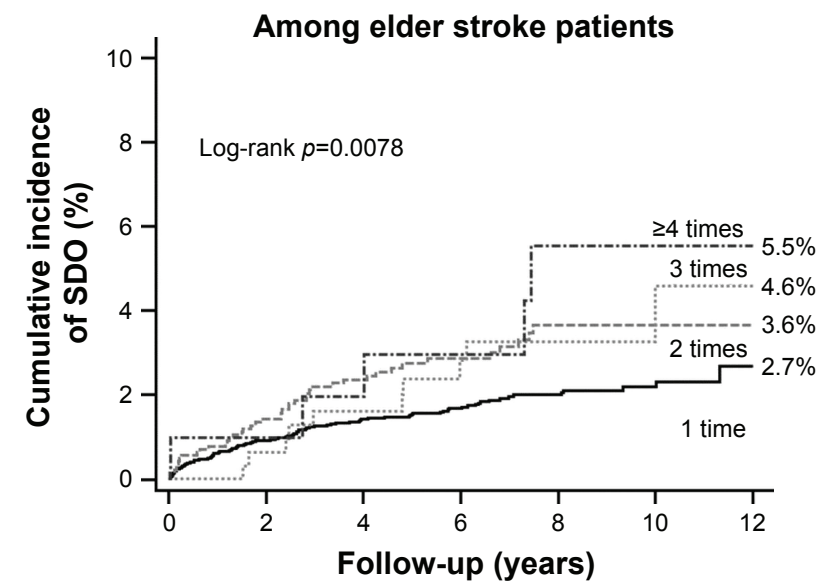

Figure 3 Cumulative incidence of SDO in elder stroke patients with different stroke attacks.

Abbreviation: SDO, suicidal drug overdose.

4) the poststroke depression group had the highest risk of SDO; 5) most SDO events occurred within 1 year following stroke in older patients with stroke $(66 / 191,34.6 \%)$; and 6) benzodiazepines and anticoagulants were common drugs for suicide. Additionally, of the two stroke types, patients with ischemic stroke had a higher risk of SDO than those with hemorrhagic stroke.

In the present study, we found that older patients with stroke had a higher risk of SDO compared with those without stroke (aHR: 2.288, 95\% CI: 1.746-2.999, $p<0.001$ ). In Sweden, Eriksson et al investigated 220,336 patients with stroke and found 1,217 suicide attempts from 2001 to 2012. The most common method of these suicide attempts was self-poisoning $(889 / 1,217,73.0 \%) .{ }^{29}$ In Korea, Chung et al enrolled 4,560 patients with stroke and 224,175 controls without stroke. Patients with stroke had a higher risk of attempting suicide (odds ratio: $1.64,95 \%$ CI: $1.21-2.22$ ) ${ }^{36}$ Furthermore, we found that the risk of SDO significantly increased with the number of stroke events. Older patients with more than four strokes had the highest cumulative incidence of SDO (Figure 3). Kishi et a ${ }^{10}$ enrolled 301 patients with acute stroke and found that stroke patients with previous stroke events had a higher rate of suicidal thoughts than those without ( $43 \%$ vs $17.7 \%$ ).

Many studies have linked depression to an increased suicide risk in patients with stroke. ${ }^{9,10,17,28}$ Kishi and Kosier ${ }^{9}$ reported that among a suicidal group $(\mathrm{n}=20), 15(75 \%)$ patients had major depression and three (15\%) had minor depression; by contrast, among a nonsuicidal group $(\mathrm{n}=281)$, $42(15 \%)$ patients had major depression and $51(18.1 \%)$ had minor depression. In our study, the stroke group had a higher rate of depressive disorder than the control group $(9.2 \%$ vs $7.7 \%$ ). Moreover, depressive disorder was an independent risk factor for SDO (aHR: 1.543, 95\% CI: 1.001-2.378,

Table 3 Univariate and multivariate survival analyses for factors associated with SDO in patients with stroke

\begin{tabular}{|c|c|c|c|c|c|c|}
\hline \multirow[t]{2}{*}{ Variables } & \multicolumn{3}{|c|}{ Univariate analysis } & \multicolumn{3}{|c|}{ Multivariate analysis } \\
\hline & HR & $95 \% \mathrm{Cl}$ of $\mathrm{HR}$ & $p$-value & aHR & $95 \% \mathrm{Cl}$ of aHR & $p$-value \\
\hline Age (years) & 1.007 & $0.989-1.025$ & 0.473 & 1.000 & $0.98 \mathrm{I}-1.020$ & 0.992 \\
\hline \multicolumn{7}{|l|}{ Sex } \\
\hline Men & 1.000 & & & & & \\
\hline Women & 0.988 & $0.743-1.313$ & 0.933 & 0.975 & $0.718-1.323$ & 0.869 \\
\hline \multicolumn{7}{|l|}{ Mental health diagnosis } \\
\hline Insomnia & 1.244 & $0.899-1.722$ & 0.188 & 1.101 & $0.78 \mathrm{I}-1.552$ & 0.584 \\
\hline Depressive disorders & 1.735 & $1.155-2.607$ & 0.008 & 1.543 & $1.00 \mathrm{I}-2.378$ & 0.050 \\
\hline Dementia & 1.522 & $0.966-2.397$ & 0.070 & 1.356 & $0.84 I-2.189$ & 0.212 \\
\hline Substance use disorders & 0.825 & $0.205-3.324$ & 0.787 & 0.846 & $0.191-3.743$ & 0.825 \\
\hline Alcohol use disorders & 0.875 & $0.280-2.738$ & 0.819 & 0.938 & $0.278-3.169$ & 0.919 \\
\hline Bipolar disorders & 2.018 & $0.50 I-8.130$ & 0.323 & 1.325 & $0.318-5.522$ & 0.699 \\
\hline Psychotic disorders & 2.130 & $0.529-8.578$ & 0.288 & 1.662 & $0.402-6.865$ & 0.483 \\
\hline \multicolumn{7}{|l|}{ Major coexisting diseases } \\
\hline Hypertension & 0.994 & $0.643-1.537$ & 0.978 & 0.882 & $0.563-1.383$ & 0.584 \\
\hline Diabetes & 1.179 & $0.887-1.568$ & 0.256 & 1.227 & $0.909-1.657$ & 0.181 \\
\hline Coronary disease & 1.614 & $1.213-2.147$ & 0.001 & 1.617 & $1.193-2.913$ & 0.002 \\
\hline COPD & 1.254 & $0.944-1.665$ & 0.119 & 1.267 & $0.912-1.762$ & 0.158 \\
\hline Chronic kidney disease & 0.850 & $0.593-1.217$ & 0.375 & 0.748 & $0.515-1.088$ & 0.128 \\
\hline Asthma & 0.952 & $0.645-1.406$ & 0.806 & 0.749 & $0.483-1.160$ & 0.195 \\
\hline Fracture & 0.897 & $0.630-1.276$ & 0.544 & 0.892 & $0.615-1.295$ & 0.549 \\
\hline Osteoporosis & 0.860 & $0.596-1.242$ & 0.422 & 0.798 & $0.532-1.198$ & 0.276 \\
\hline Hyperlipidemia & 0.877 & $0.617-1.247$ & 0.466 & 0.798 & $0.554-1.148$ & 0.223 \\
\hline
\end{tabular}

Abbreviations: SDO, suicidal drug overdose; $\mathrm{HR}$, hazard ratio; $\mathrm{Cl}$, confidence interval; a HR, adjusted hazard ratio; COPD, chronic obstructive pulmonary disease. 


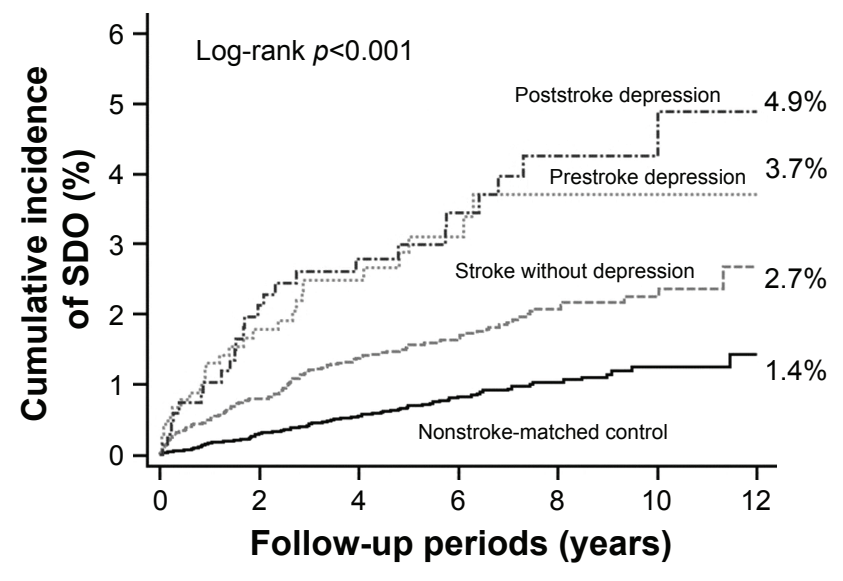

Figure 4 Cumulative incidence of SDO in four groups: poststroke depression, prestroke depression, stroke without depression, and nonstroke-matched control groups.

Abbreviation: SDO, suicidal drug overdose.

$p=0.050$ ), and the poststroke depression group had the highest risk of SDO (Figure 4). A study on 2,283 suicide victims during 1988-2007 in northern Finland reported that $70 \%$ of patients with prestroke depression committed suicide within 2 years after stroke. ${ }^{21}$ In our study, $54.3 \%$ of older patients with stroke developed SDO within 2 years after stroke.

In this study, we found that benzodiazepines and anticoagulants were common drugs used for SDO. Benzodiazepines are medications commonly prescribed for anxiety, agitation, insomnia, and alcohol withdrawal. A study in the United States during 2001-2010 revealed that benzodiazepines were used in 16.6 million of 133.3 million ambulatory clinic visits and benzodiazepine use in individuals at the

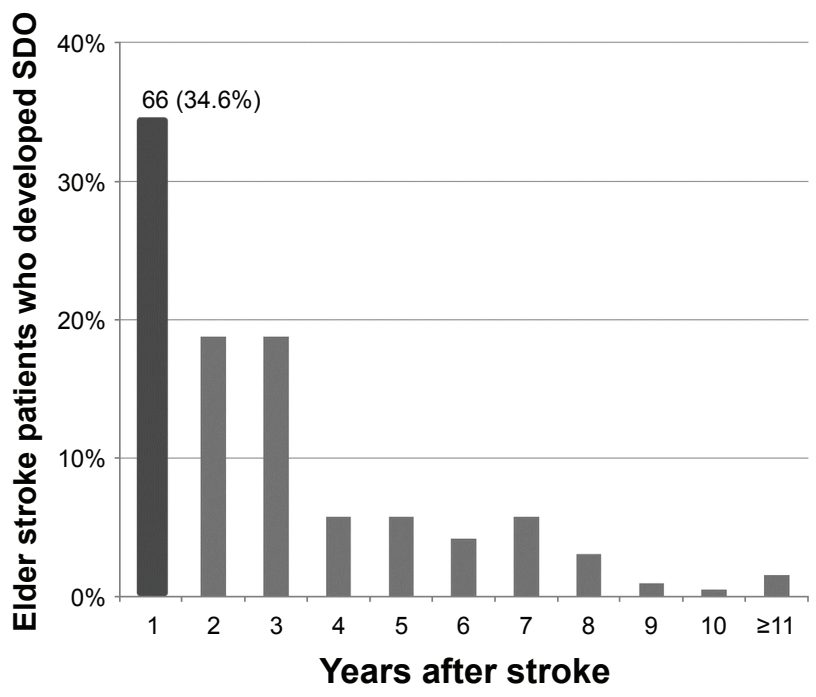

Figure 5 Follow-up duration among 191 elder stroke patients who developed SDO. Abbreviation: SDO, suicidal drug overdose. age of 85 years or older increased from $8.9 \%$ to $19.3 \% .{ }^{37} \mathrm{~A}$ study linked benzodiazepines with the risk of suicide and suicide attempts (odds ratio: 2.08, 95\% CI: 1.83-2.36). ${ }^{35}$ Regarding anticoagulants, Placido and Sposito reported that the incidence of deaths from suicide was positively correlated with that of deaths from ischemic heart disease $(r=0.95$, $p<0.0001) .{ }^{26}$ Anticoagulants are commonly prescribed for ischemic heart disease (coronary disease), ${ }^{38}$ which is a common comorbidity of stroke. This suggests an association between anticoagulants and SDO in geriatric patients with stroke. Further research is required to investigate the details of medication use.

Various mechanisms may underlie the association between stroke and SDO in older patients. First, previous studies have reported that patients with stroke usually use nonviolent suicide methods., ${ }^{4,21}$ A study in Sweden found 1,217 suicide attempts out of 220,336 patients with stroke, and the leading method of these suicide attempts was self-poisoning $(889 / 1,217,73.0 \%){ }^{29}$ This may result from the increased use of medications in older people with comorbidities ${ }^{39}$ and the disability caused by stroke. ${ }^{2}$ Second, stroke may cause stress and disability in older patients, ${ }^{2}$ which may result in a high incidence of poststroke depression, ranging from $20 \%$ to $50 \%$, according to data from self-report questionnaires. ${ }^{40}$ Depressed patients have a higher risk of suicide. ${ }^{41}$ Third, previous studies have found that stroke may impair the frontal-subcortical circuit and neuropsychological function, thereby increasing impulsivity. ${ }^{12,42}$ This may also contribute to the increased risk of drug overdose in older patients with stroke.

This study had several limitations. First, we identified SDO or self-poisoning on the basis of ICD codes. ${ }^{33-35}$ In East Asia, people tend to stigmatize suicide and deny suicide attempts. ${ }^{43,44}$ Additionally, patients with minor drug overdose may not seek medical services. Therefore, the incidence of SDO may be higher. Second, NHIRD does not provide information on genetic or psychosocial factors, which are potential confounders associated with the risk of SDO. Third, details on other methods of suicide attempt, patients who committed suicide, and medications are not available in our database.

\section{Conclusion}

Older patients aged 60-99 years with stroke had a higher risk of SDO compared with those without stroke, and the risk was increased in those with depressive disorder, coronary disease, and ischemic stroke. We found that most SDO events occurred within 1 year following stroke in older patients 
with stroke (66/191, 34.6\%). Moreover, benzodiazepines and anticoagulants were the two most common drugs used for SDO. These results strongly suggest that psychological assessment and medication monitoring should be incorporated into current neurology clinical diagnoses and treatments for older patients with stroke.

\section{Acknowledgments}

This study is supported, in part, by the Taiwan Ministry of Health and Welfare Clinical Trial Center (MOHW105-TDUB-212-133019). The funding agencies did not influence the study design, data collection or analysis, decision to publish, or preparation of the manuscript.

\section{Author contributions}

Chun-Hung Chang proposed the research ideas, conducted statistical analysis, performed database processing, and drafted the initial manuscript. Shaw-Ji Chen and Chieh-Yu Liu applied for the database and provided expert opinions. Hsin-Chi Tsai supervised the study. All authors contributed toward data analysis, drafting and critically revising the paper and agree to be accountable for all aspects of the work.

\section{Disclosure}

The authors report no conflicts of interest in this work.

\section{References}

1. Sacco RL, Kasner SE, Broderick JP, et al. An updated definition of stroke for the 21 st century: a statement for healthcare professionals from the American Heart Association/American Stroke Association. Stroke. 2013;44(7):2064-2089.

2. Hankey GJ. Stroke. Lancet. 2017;389(10069):641-654.

3. Patel AT. Disability evaluation following stroke. Phys Med Rehabil Clin N Am. 2001;12(3):613-619.

4. Pompili M, Venturini P, Lamis DA, et al. Suicide in stroke survivors: epidemiology and prevention. Drugs Aging. 2015;32(1):21-29.

5. Pompili M, Venturini P, Campi S, et al. Do stroke patients have an increased risk of developing suicidal ideation or dying by suicide? An overview of the current literature. CNS Neurosci Ther. 2012;18(9): 711-721.

6. Bartoli F, Pompili M, Lillia N, et al. Rates and correlates of suicidal ideation among stroke survivors: a meta-analysis. J Neurol Neurosurg Psychiatry. 2017;88(6):498-504.

7. Park SM. Health status and suicidal ideation in Korean elderly: the role of living arrangement. J Ment Health. 2014;23(2):94-98.

8. Shin KM, Cho SM, Hong CH, et al. Suicide among the elderly and associated factors in South Korea. Aging Ment Health. 2013;17(1): 109-114.

9. Kishi Y, Kosier JT, Robinson RG. Suicidal plans in patients with acute stroke. J Nerv Ment Dis. 1996;184(5):274-280.

10. Kishi Y, Robinson RG, Kosier JT. Suicidal plans in patients with stroke: comparison between acute-onset and delayed-onset suicidal plans. Int Psychogeriatr. 1996;8(4):623-634.

11. Pohjasvaara T, Vataja R, Leppavuori A, Kaste M, Erkinjuntti T. Suicidal ideas in stroke patients 3 and 15 months after stroke. Cerebrovasc Dis. 2001;12(1):21-26.
12. Rao R, Jackson S, Howard R. Neuropsychological impairment in stroke, carotid stenosis, and peripheral vascular disease, a comparison with healthy community residents. Stroke. 1999;30(10):2167-2173.

13. Santos CO, Caeiro L, Ferro JM, Figueira ML. A study of suicidal thoughts in acute stroke patients. J Stroke Cerebrovasc Dis. 2012; 21(8):749-754.

14. Fuller-Thomson E, Tulipano MJ, Song M. The association between depression, suicidal ideation, and stroke in a population-based sample. Int J Stroke. 2012;7(3):188-194.

15. Tang WK, Lu JY, Liang H, et al. Is insomnia associated with suicidality in stroke? Arch Phys Med Rehabil. 2011;92(12):2025-2027.

16. Tang WK, Lu JY, Mok V, Ungvari GS, Wong KS. Is fatigue associated with suicidality in stroke? Arch Phys Med Rehabil. 2011;92(8): 1336-1338.

17. Chan SS, Lyness JM, Conwell Y. Do cerebrovascular risk factors confer risk for suicide in later life? A case-control study. Am J Geriatr Psychiatry. 2007;15(6):541-544.

18. Yamauchi $\mathrm{T}$, Inagaki $\mathrm{M}$, Yonemoto $\mathrm{N}$, et al. Death by suicide and other externally caused injuries after stroke in Japan (1990-2010): the Japan Public Health Center-based prospective study. Psychosom Med. 2014;76(6):452-459.

19. Stenager EN, Madsen C, Stenager E, Boldsen J. Suicide in patients with stroke: epidemiological study. BMJ. 1998;316(7139):1206.

20. Teasdale TW, Engberg AW. Suicide after a stroke: a population study. J Epidemiol Community Health. 2001;55(12):863-866.

21. Forsstrom E, Hakko H, Nordstrom T, Rasanen P, Mainio A. Suicide in patients with stroke: a population-based study of suicide victims during the years 1988-2007 in northern Finland. J Neuropsychiatry Clin Neurosci. 2010;22(2):182-187.

22. Katayama M, Naritomi H, Oomura M, et al. Case reports of unexpected suicides in patients within six months after stroke. Kobe J Med Sci. 2011;56(5):E184-E194

23. Garden FH, Garrison SJ, Jain A. Assessing suicide risk in stroke patients: review of two cases. Arch Phys Med Rehabil. 1990;71(12): 1003-1005.

24. Scott KM, Hwang I, Chiu WT, et al. Chronic physical conditions and their association with first onset of suicidal behavior in the world mental health surveys. Psychosom Med. 2010;72(7):712-719.

25. Voaklander DC, Rowe BH, Dryden DM, Pahal J, Saar P, Kelly KD Medical illness, medication use and suicide in seniors: a populationbased case-control study. J Epidemiol Community Health. 2008;62(2): $138-146$.

26. Placido A, Sposito AC. Association between suicide and cardiovascular disease: time series of 27 years. Int J Cardiol. 2009;135(2):261-262.

27. Webb RT, Kontopantelis E, Doran T, Qin P, Creed F, Kapur N. Suicide risk in primary care patients with major physical diseases: a case-control study. Arch Gen Psychiatry. 2012;69(3):256-264.

28. Crump C, Sundquist K, Sundquist J, Winkleby MA. Sociodemographic, psychiatric and somatic risk factors for suicide: a Swedish national cohort study. Psychol Med. 2014;44(2):279-289.

29. Eriksson M, Glader EL, Norrving B, Asplund K. Poststroke suicide attempts and completed suicides: a socioeconomic and nationwide perspective. Neurology. 2015;84(17):1732-1738.

30. Mann JJ, Marzuk PM, Arango V, McBride PA, Leon AC, Tierney H. Neurochemical studies of violent and nonviolent suicide. Psychopharmacol Bull. 1989;25(3):407-413.

31. Hawton K, van Heeringen K. Suicide. Lancet. 2009;373(9672): 1372-1381.

32. Chan HL, Liu CY, Chau YL, Chang CM. Prevalence and association of suicide ideation among Taiwanese elderly - a population-based cross-sectional study. Chang Gung Med J. 2011;34(2):197-204.

33. Tang KT, Lin $\mathrm{CH}$, Chen $\mathrm{HH}$, Chen YH, Chen DY. Suicidal drug overdose in patients with systemic lupus erythematosus, a nationwide population-based case-control study. Lupus. 2016;25(2):199-203.

34. Chou IC, Lin CC, Sung FC, Kao CH. Attention-deficit hyperactivity disorder increases the risk of deliberate self-poisoning: a populationbased cohort. Eur Psychiatry. 2014;29(8):523-527. 
35. Shih HI, Lin MC, Lin CC, et al. Benzodiazepine therapy in psychiatric outpatients is associated with deliberate self-poisoning events at emergency departments-a population-based nested case-control study. Psychopharmacology (Berl). 2013;229(4):665-671.

36. Chung JH, Kim JB, Kim JH. Suicidal ideation and attempts in patients with stroke: a population-based study. J Neurol. 2016;263(10):2032-2038.

37. Marra EM, Mazer-Amirshahi M, Brooks G, van den Anker J, May L, Pines JM. Benzodiazepine prescribing in older adults in U.S. Ambulatory Clinics and Emergency Departments (2001-2010). J Am Geriatr Soc. 2015;63(10):2074-2081.

38. Cove CL, Hylek EM. An updated review of target-specific oral anticoagulants used in stroke prevention in atrial fibrillation, venous thromboembolic disease, and acute coronary syndromes. J Am Heart Assoc. 2013;2(5):e000136.

39. Cobaugh DJ, Miller MJ, Pham TT, Krenzelok EP. Risk of major morbidity and death in older adults with suicidal intent: a cross-sectional analysis from the National Poison Data System, 2000-2009. J Am Geriatr Soc. 2015;63(3):501-507.
40. Morris PL, Robinson RG, Raphael B. Prevalence and course of depressive disorders in hospitalized stroke patients. Int J Psychiatry Med. 1990;20(4):349-364.

41. Belmaker RH, Agam G. Major depressive disorder. $N$ Engl J Med. 2008;358(1):55-68.

42. Vataja R, Pohjasvaara T, Mantyla R, et al. Depression-executive dysfunction syndrome in stroke patients. Am J Geriatr Psychiatry. 2005;13(2):99-107.

43. Chen JA, Courtwright A, Wu KC. The role of stigma and denormalization in suicide-prevention laws in East Asia: a sociocultural, historical, and ethical perspective. Harv Rev Psychiatry. 2017;25(5):229-240.

44. Carpiniello B, Pinna F. The reciprocal relationship between suicidality and stigma. Front Psychiatry. 2017;8:35.

\section{Publish your work in this journal}

Neuropsychiatric Disease and Treatment is an international, peerreviewed journal of clinical therapeutics and pharmacology focusing on concise rapid reporting of clinical or pre-clinical studies on a range of neuropsychiatric and neurological disorders. This journal is indexed on PubMed Central, the 'PsycINFO' database and CAS, and is the official journal of The International Neuropsychiatric Association (INA). The manuscript management system is completely online and includes a very quick and fair peer-review system, which is all easy to use. Visit http://www.dovepress.com/testimonials.php to read real quotes from published authors.

Submit your manuscript here: http://www.dovepress.com/neuropsychiatric-disease-and-treatment-journal 